\title{
C5orf30 regulates severity of tissue destruction in RA
}

Chromosome 5 open reading frame 30 (C5orf30) has been genetically associated with risk of rheumatoid arthritis (RA) and with severity of joint damage in patients with the disease. Although expression of this gene is highly conserved in vertebrate genomes, little is known about the biological function of human C5orf30. A group of researchers from University College Dublin and the University of Sheffield now report that C5orf30 is a previously unidentified negative regulator of tissue damage in RA, through its effects on the autoaggressive phenotype of synovial fibroblasts (RASFs).

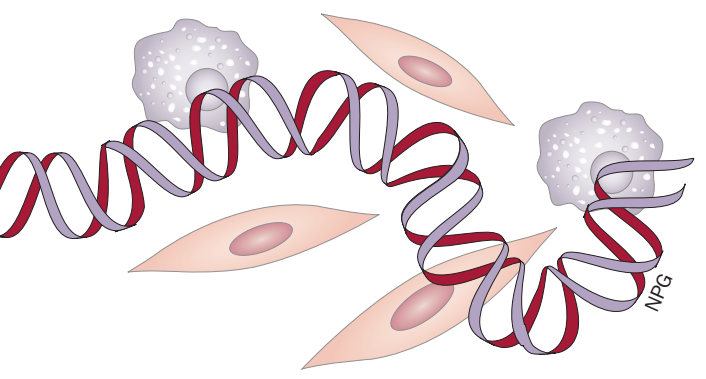

Munitta Muthana and colleagues showed that C5orf30 mRNA is expressed at high levels in the synovium of patients with RA (relative to healthy individuals or those with osteoarthritis), predominantly in RASFs and synovial macrophages. In vitro, exposure of cultured RASFs to hypoxia $\left(0.5 \% \mathrm{O}_{2}\right)$ induced an eightfold increase in expression of C5orf30, whereas treatment with TNF led to reduced (0.5-fold) C5orf30 expression; C5orf30 expression was also decreased by the combination of TNF and hypoxia.

Knockdown of C5orf30 expression using small interfering RNA (siRNA) led to increased migration and invasiveness of RASFs - characteristics linked to the joint-damaging potential of these cells. Moreover, C5orf30 affected RASF expression of $>100$ arthritis-related genes involved in cell adhesion and migration, the inflammatory response, angiogenesis, as well as extracellular matrix proteins.

Based on the results of the RASF experiments, the researchers hypothesized that inhibition of C5orf30 might drive inflammation and tissue destruction in a mouse model of RA. Accordingly, collageninduced arthritis was exacerbated in mice treated with C5orf30 siRNA in comparison with mice treated with nontargeted control siRNA or vehicle. C5orf30-knockdown mice had more severe inflammation, bone erosion and cartilage degradation than the other groups, in parallel with increased expression of TNF and IL-1 and decreased expression of anti-inflammatory IL-10 in the joints.

"These exciting findings will prompt us to further explore the role of this highly conserved protein that we know so little about," says Muthana. "Our findings also provide a genetic marker that could be used to identify those RA patients who require more aggressive treatments or personalized medicine."

\section{Sarah Onuora}

Original article Muthana, M. et al. C5orf30 is a negative regulator of tissue damage in rheumatoid arthritis. Proc. Natl Acad. Sci. USA 112, 11618-11623 (2015) 